schied sich nach mehreren Stunden ein gelbliches Pulver, Wolframsäure, ab.

Stettin, den 1. August 1869.

\title{
Zur Bestimmung des Urans.
}

Von

\section{Dr. Cl. Winkler.}

Bei Durchführung einer technischen Arbeit über die Gewinnung des Urans aus seinen Erzen, fand ich Gelegenheit, die von A. Patera angegebene Uranprobe ${ }^{*}$ ) vielfach anzuwenden, und dieselbe einer Prüfung zu unterwerfen. Es hat sich dabei ergeben, dass diese Methode sich nicht allein durch Kürze und Bequemlichkeit empfiehlt, sondern. dass sie auch richtige und mit der Analyse so nahe übereinstimmende Resultate liefert, dass man sie unbedenklich als mindestens für die Technik linreichend genau bezeichnen kann. Etwas zu hohe Resultate gibt sie bei starkem Kupfergehalt der antersuchten Erze, wo dann jederzeit eine geringe Menge Kupfer mit in die alkalische Lösung übergeht und beim nachherigen Zusatz von Aetznatron wieder mit ausgefällt wird.

In $\mathrm{Bd}$. V, p. 228 dieser Zeitschrift findet sich ein Referat über die Patera'sche Uranprobe, in welchem unter Anderem gesagt ist:

"Nach Angabe des Verfassers entsprechen 100 Thl. des UranoxydNatrons 88,3 Thl. Uranoxydoxydul. Ich bemerke hierzu, dass Patera hierunter nicht das gewöhnliche Uranoxydoxydul verstehen kann, sondern die von Pélig ot beschriebene Verbindung $\mathrm{U}_{4} \mathrm{O}_{5}=2 \mathrm{UO}, \mathrm{U}_{2} \mathrm{O}_{3}$, denn 100 Thl. $\mathrm{NaO}, 2 \mathrm{U}_{2} \mathrm{O}_{3}$ entsprechen 36,39 Thl. $\mathrm{U}_{3} \mathrm{O}_{4}{ }^{* *}$ ) und 87,68 Thl. $\mathrm{U}_{4} \mathrm{O}_{5}$. Richtiger wird es sein, sich bei der Berechnung des letzteren Werthes zu bedienen, welchem das Aequivalentgewicht 59,4 (nach Ebelmen) zu Grunde liegt. Diese Zahl hat bekanntlich Berzelius schon für die richtigere gehalten, und sie erlangt eine Bestätigung durch die

*) Dingl. polyt. Journ. 180. p. 242.

**) Diese Zahl seheint verdruckt zu sein und soll wohl heissen $66,39 \mathrm{Thl}$. $\mathrm{U}_{3} \mathrm{O}_{4}$. 
Analysen des phosphorsauren Uranoxyds, welche von Knop und Arendt ausgeführt worden sind, die bei Annahme des von, Ebelmen bestimmten Aequivalents besser mit der theoretischen Menge stimmen, als bei der Zugrundelegung der Zahl 60 , wie sie von Pélig ot ermittelt wurde."

Dieser Einwurf beruht auf einem Irrthum. Ich vermuthe, dass die Berechnungsweise, aus der er hervorgegangen, folgende ist:

und :

1) $\mathrm{NaO}_{2}, \mathrm{U}_{2} \mathrm{O}_{3}: \mathrm{U}_{3} \mathrm{O}_{4}=100: \mathrm{x}$. $316,6 \quad: 210,2=100: \mathrm{x}$.

$$
66,39=\mathrm{x} \text {. (angegeben 36,39). }
$$

2) $\mathrm{NaO}, 2 \mathrm{U}_{2} \mathrm{O}_{8}: \mathrm{U}_{4} \mathrm{O}_{5}=100: \mathrm{x}$.

$316,6: 277,6=100: \mathrm{x}$.

$87,68=\mathrm{x}$.

Im ersteren Falle ist also fälschlich gesetzt.

$$
\mathrm{NaO}, 2 \mathrm{U}_{2} \mathrm{O}_{3} \text { entsprechend } \mathrm{U}_{3} \mathrm{O}_{4} \text { oder } \mathrm{U}_{4}=\mathrm{U}_{3}
$$

In Wirklichkeit entsprechen

$$
\begin{aligned}
3\left(\mathrm{Na} \mathrm{O}, 2 \mathrm{U}_{2} \mathrm{O}_{3}\right) & =4\left(\mathrm{U}_{3} \mathrm{O}_{4}\right) \text { oder } \\
& =3\left(\mathrm{U}_{4} \mathrm{O}_{5}\right)
\end{aligned}
$$

und der procentale Gehalt des sauren uransauren Natrons an Uranoxydoxydul würde betragen bei Annahme von

$$
\begin{aligned}
& \mathrm{U}_{3} \mathrm{O}_{4}=88,52 \\
& \mathrm{U}_{4} \mathrm{O}_{5}=87,68 .
\end{aligned}
$$

Es liegt mithin kein Grund vor, anzunehmen, dass Patera sich eines zu hohen Aequivalents und einer falschen Formel für das Uranoxydoxydul bedient habe; wahrscheinlicher ist es, dass die von ihm gemachte Gehaltsangabe von $88,3 \%$ Uranoxydoxydul im sauren uransauren Natron das Resultat directer analytischer Untersuchung der letztgenannten Verbindung ist.

Directe Bestimmung der Baldriansäure in den baldriansauren Salzen.

\section{Von \\ Antonio Zavatti und Fausto Sestini,}

Die directe Bestimmung der Baldriansäure in ihren, in der Pharmacie angewendeten, Salzen bietet keine geringe Schwierigkeit dar; aber wir 